\title{
Analisi dei costi di buprenorfina vs metadone nella terapia dei soggetti con dipendenza da oppiacei
}

Giorgio L. Colombo, Guido Faillace, Michele Ferdico

\begin{abstract}
In the last decades, methadone has virtually represented the only available option for the treatment of opioid addicts in Italy. Early in the year 2000 buprenorphine has been introduced on the market as a possible alternative to methadone. While most of the research conducted so far in our country has focused on possible differences in clinical outcomes, depending on the pharmacological differences among the two molecules, the economical aspects of the choice between the two drugs have barely been addressed. In this paper we present a pharmacoeconomical comparison between buprenorphine and methadone in the treatment of heroin dependence by the Italian health service, adopting the perspective of the Society.

For this purpose, we constructed a decision analysis model comprising the possible clinical pathways a patient can go through following the inclusion in the therapeutic programs of the SerT. Two types of incremental costs have been considered in the model: direct health costs, i.e. drug acquisition and medical staff costs, and indirect cost, due to loss of working hours for the administration of the drug. The analysis was conducted with a cost-minimization approach, mandatory in the absence of reliable data on clinical differences, and with a time perspective of 12 months.

Our results indicate that buprenorphine, despite its higher pharmaceutical cost, is the more convenient option for the treatment of opioid dependence in Italy, as it permits a significant saving of productivity losses. The difference in indirect costs relies on the shorter mean treatment time with buprenorphine and on the possibility of reducing dosing frequency, which allows many patients to attend health services only thrice in week, instead of the daily visits to the SerT necessary for most methadone-treated patients. The results were challenged in a series of sensibility analysis conducted on all relevant and uncertain paramenters, and in no case the option methadone resulted more convenient than buprenorphine, confirming the reliability of the results obtained in the base case scenario.
\end{abstract}

Farmeconomia e percorsi terapeutici 2003; 4 (4): 185-192

\section{INTRODUZIONE}

In Italia il trattamento della tossicodipendenza, a causa soprattutto della scarsa efficacia delle alternative di trattamento proposte, è stato contraddistinto a partire dagli ultimi decenni dall'impiego di metadone (Met) quale principale, se non unico, presidio disponibile [1]. Infatti sia le tecniche UROD (detossificazione ultrarapida da oppiacei) sia quelle rapide (day hospital o degenza ospedaliera per 5-7 giorni) sono state caratterizzate da un'elevata percentuale di recidive, mentre l'introduzione di farmaci alternativi come il naltrexone si è rilevata più utile verso quei pazienti che avevano già raggiunto uno stato di drug free che per la detossificazione [2]. È in questo contesto che il metadone si è affermato come terapia di prima scelta nel trattamento dell'eroinodipendenza.

Il metadone è un agonista completo dei recettori cerebrali per gli oppioidi. A dosaggi adeguati al livello di dipendenza è in grado di soddisfare la richiesta compulsiva della sostanza di abuso interrompendo così l'uso di oppioidi.

La buprenorfina, introdotta nei primi mesi del 2000 nel mercato italiano, è un agonista parziale dei recettori mu, che è in grado di coprire il bisogno compulsivo di sostanze fino ad annullare l'uso, ma senza raggiungere gli effetti collaterali caratteristici degli agonisti completi, quali depressione respiratoria signifi-
S.A.V.E.

Studi Analisi

Valutazioni

Economiche, Milano 
cativa e alterazione delle risposte e dell'espressione recettoriale. Tutto ciò si traduce in un'elevata sicurezza e un basso livello di dipendenza.

L'altra peculiarità della buprenorfina è la sua elevata affinità recettoriale verso i sistemi mu e kappa. Nei confronti del primo si ha il blocco dei recettori sottraendoli all'azione euforizzante dell'eroina assunta nelle ricadute. Nel sistema kappa invece, verso il quale si ha il solo blocco del recettore senza stimolarlo (antagonismo), la buprenorfina esercita un' azione anti-disforizzante e anti-depressiva in ragione di una sovra espressione dell'agonista endogeno dinorfina, tipica di chi fa un uso cronico di sostanze in grado di dare reward [6,7].

Attualmente, a distanza di tre anni dall'introduzione di buprenorfina (Bpn), si è affermato un nuovo scenario con il quale gli operatori dei Servizi per le Tossicodipendenze (Ser.T.) si possono confrontare: la possibilità di valutare l'utilizzo di due terapie valide e confrontabili nel trattamento dell'eroinodipendenza: buprenorfina e metadone.

La relativa letteratura scientifica italiana si è per ora soffermata in prevalenza sugli aspetti clinici, indagando le differenze tra i due farmaci in termini di effectiveness, di adverse drug reaction e di interazione con altre terapie farmacologiche (anti-Hiv, anti-HCV) spesso prescritte ai soggetti tossicodipendenti [1].

L'obiettivo di questo studio è quello di confrontare i costi sanitari (diretti) e le perdite di produzione (costi indiretti) delle due terapie farmacologiche, buprenorfina e metadone, impiegate nel trattamento della dipendenza da oppiacei, al fine di valutare il loro impatto economico sul sistema sanitario e sulla società.

\section{METODIESTRUMENTI}

\section{Premessa}

La presente valutazione economica ha come oggetto di studio la comparazione dei costi di due terapie farmacologiche, buprenorfina e metadone, impiegate nel trattamento della tossicodipendenza.

La valutazione dei costi, assumendo come prospettiva di riferimento quella propria della Società, si è sviluppata lungo la logica di minimizzazione dei costi (cost-minimization) [8].

Nel modello di valutazione sono stati inclusi sia i costi diretti, cioè quelli legati al costo di acquisto e alla somministrazione delle terapie, sia quelli indiretti (o costi sociali) connessi alle eventuali perdite di giornate (o ore) di lavoro. L'orizzonte temporale di riferimento dell'analisi dei costi è di 12 mesi. L'anno di riferimento per la stima dei costi per il trattamento dell'eroinodipendenza è il 2003. Vale a dire che i consumi sono stati valorizzati con il medesimo parametro monetario. In questo modo, $i$ costi per il trattamento della tossicodipendenza si differenziano in base al numero e alla tipologia di prestazioni erogate.

\section{Modello}

L'analisi di minimizzazione dei costi è stata effettuata mediante la predisposizione ad hoc di un modello decisionale con struttura ad albero [10]. Tale modello è rappresentato nella Figura 1.

I soggetti tossicodipendenti entrano alla radice dell'albero decisionale e seguono uno dei possibili scenari (da sinistra verso destra).
Figura 1

Albero decisionale: buprenorfina (Bpn) vs metadone (Met)

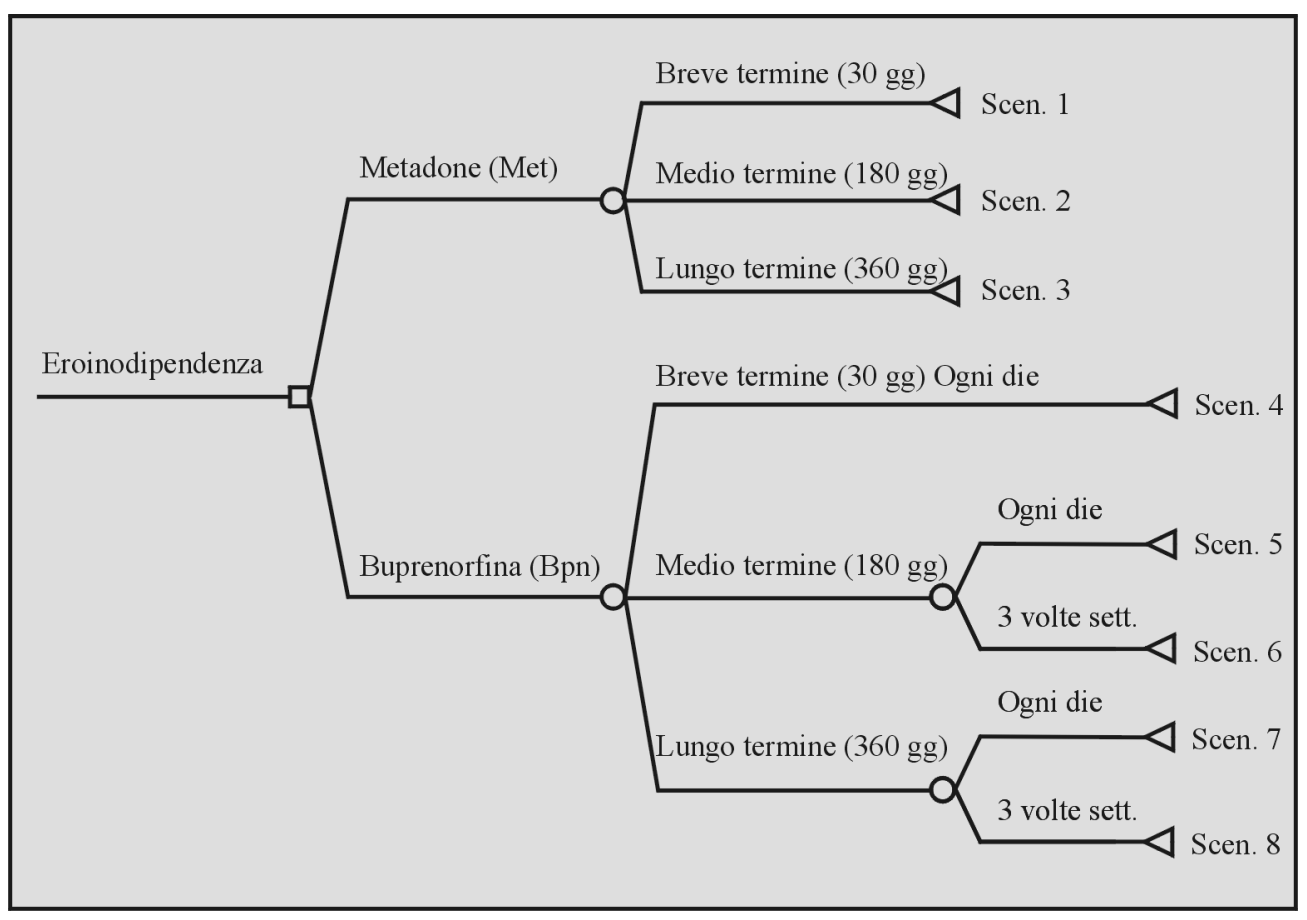


Il primo passo del modello è quello di assegnare i soggetti ad uno dei due trattamenti, metadone o buprenorfina.

Braccio metadone. Entro l'arco temporale considerato nella valutazione economica, cioè quello di un anno, i soggetti hanno una specifica probabilità di terminare il trattamento entro tre definiti periodi:

i) breve termine $(\mathrm{t}=<30 \mathrm{gg})$,

ii) medio termine $(30 \mathrm{gg}<\mathrm{t}=<180 \mathrm{gg})$,

iii) lungo termine $(\mathrm{t}<360 \mathrm{gg})$.

Sia per il breve che per il medio termine è prevista una somministrazione giornaliera del farmaco da effettuarsi presso i Ser.T. Nel lungo termine (12 mesi), è stata considerata la possibilità di passare nella seconda metà dell'anno ad un regime di parziale affido (fino ad un massimo di 7 giorni) del farmaco per migliorare la compliance del paziente.

Braccio buprenorfina. Anche per i soggetti trattati con buprenorfina si presenta la possibilità di terminare il trattamento entro gli stessi definiti periodi, ma con probabilità di accadimento differenti. In questo braccio infatti sono state previste delle varianti che differenziano i possibili percorsi dei soggetti trattati rispetto al metadone. Dato che una delle principali caratteristiche di buprenorfina è la possibilità di essere somministrata in una unica soluzione a dosaggi multiplii rispetto a quello giornaliero (permettendo così ai soggetti di presentarsi in media 3 volte alla settimana nei Ser.T. per ricevere la terapia), nel modello è stata considerata la possibilità che parte dei soggetti trattati con buprenorfina, sia nel medio che nel lungo termine, passi a questa modalità di dosaggio multiplo [11].

I primi 30 giorni di terapia presentano delle caratteristiche comuni per entrambi i trattamenti. L'impiego del personale medico è più concentrato nei primi giorni essenzialmente per il monitoraggio della terapia e dei suoi esiti intermedi, mentre quello del personale infermieristico diventa maggiormente rilevante solo dopo la stabilizzazione del paziente ad un dosaggio del trattamento giudicato efficace. Sia per buprenorfina che per metadone in questi primi 30 giorni le somministrazioni sono a dosi singole ed effettuate giornalmente nei Ser.T.

Dato che per i soggetti che assumono sia Bpn sia Met, una certa quantità del proprio tempo deve essere spesa per recarsi giornalmente ai Ser.T. per ricevere le terapia, nel modello adottato per entrambi i trattamenti (buprenorfina e metadone) è stato considera- to il costo indiretto associato alla perdita di possibili ore lavorative.

Il modello è stato infine completato con appropriati valori di probabilità per definire i nodi probabilistici, e con valori puntuali di costo per espletare il confronto di cost-minimization.

\section{Probabilità degli eventi}

Le probabilità di durata del trattamento (breve, medio e lungo termine) esposte in Tabella 1 e riportate nel modello ad albero fanno riferimento a dati rilevati sull'intero territorio nazionale e pubblicati dal Ministero della Salute del 2002 [12].

In base a quanto suggerito sia dalla pratica clinica sia da numerose pubblicazioni nazionali e internazionali, dopo i primi sei mesi di somministrazione, come già ricordato, per metadone può essere prevista la possibilità che il farmaco venga affidato. In Tabella 2 viene presentata una suddivisione percentuale realistica dei soggetti trattati con metadone secondo il numero di somministrazioni settimanali. La stima di queste percentuali è stata calcolata sulla base di dati pubblicati in Convegni nazionali da diversi Ser.T. lombardi.

Infine in Tabella 3 vengono presentate le percentuali di soggetti trattati con buprenorfina che, dopo i primi trenta giorni di terapia a singole somministrazioni giornaliere effettuate nei Ser.T., passano, sia nel medio che nel lungo termine, ad una modalità tri-settimanale di assunzione del farmaco. Queste stime sono state calcolate sulla base di dati e di esperienze maturate in diversi Ser.T. lombardi. Tali percentuali sono state successivamente sottoposte all'analisi di sensibilità.

\section{Nota i}

Lo schema delle somministrazioni frazionate nella settimana prevede l'assunzione del doppio dosaggio il lunedi, seguito da un'identica somministrazione il mercoledì e dalla dispensazione del triplo dosaggio il venerdì per evitare di presentarsi al Ser.T. durante il fine settimana

\begin{tabular}{lccc}
\hline Farmaco & $\begin{array}{c}\text { Breve Termine } \\
(\mathbf{t}=<\mathbf{3 0 g g})\end{array}$ & $\begin{array}{c}\text { Medio Termine } \\
(\mathbf{3 0 g g}<\mathbf{t}=<\mathbf{1 8 0 g g})\end{array}$ & $\begin{array}{c}\text { Lungo Termine } \\
(\mathbf{t}<\mathbf{3 6 0 g g})\end{array}$ \\
\hline Metadone & $16,6 \%$ & $23,0 \%$ & 60,4 \\
Buprenorfina & $20,0 \%$ & $38,4 \%$ & 41,6 \\
\hline
\end{tabular}

\section{Tabella 1}

Probabilità dei soggetti trattati in funzione della durata della terapia

\begin{tabular}{|c|c|c|c|c|}
\hline $\begin{array}{c}\mathrm{N}^{\circ} \\
\text { somm./sett. }\end{array}$ & $\begin{array}{c}1 \\
\text { somm./sett. }\end{array}$ & $\begin{array}{c}2 \\
\text { somm./sett. }\end{array}$ & $\begin{array}{c}3 \\
\text { somm./sett. }\end{array}$ & $\begin{array}{c}7 \\
\text { somm./sett. }\end{array}$ \\
\hline Metadone & $10 \%$ & $20 \%$ & $20 \%$ & $50 \%$ \\
\hline
\end{tabular}

Tabella 2

Probabilità secondo il numero di somministrazioni settimanali (181- 360 gg) 


\section{Risorse consumate e costi unitari}

Come già ricordato, le risorse consumate $\mathrm{e}$ le relative valorizzazioni in termini monetari sono state considerate dal punto di vista della Società. I costi complessivi dei due trattamenti messi a confronto sono stati rappresentati come la somma dei costi diretti sanitari e dei costi indiretti. Si sono considerate esclusivamente le voci di costo incrementali e cioè non comuni ai due trattamenti. Nei costi diretti sanitari sono stati inclusi i costi di acquisto dei farmaci e i costi delle somministrazioni delle terapie effettuate presso i Ser.T. Nei costi indiretti si sono invece considerate le ore di lavoro perse dai soggetti in terapia per recarsi ai Ser.T. per ricevere il trattamento.

Lombardia,

Lazio e Sicilia

\begin{tabular}{lcc}
\hline Buprenorfina & $\begin{array}{c}\text { Medio Termine } \\
(\mathbf{3 0 g g}<\mathbf{t}=<\mathbf{1 8 0 g g})\end{array}$ & $\begin{array}{c}\text { Lungo Termine } \\
(\mathbf{t}<\mathbf{1 8 0 g g})\end{array}$ \\
\hline Somm. Ogni Die & $30 \%$ & $30 \%$ \\
Somm. 3 Volte Sett. & $70 \%$ & $70 \%$ \\
\hline
\end{tabular}

Tabella 3

Probabilità secondo il numero di somministrazioni settimanali, medio e lungo termine (buprenorfina)

\section{Tabella 4}

Costo medio di acquisto dei trattamenti per mg (anno 2003)

\begin{tabular}{lc}
\hline Farmaco & $\begin{array}{c}\text { Costo medio di acquisto } \\
\text { effettivo (farmacie Ser.T.) } \\
\text { (in euro) }\end{array}$ \\
\hline Metadone & 0,013 \\
Buprenorfina & 0,270 \\
\hline
\end{tabular}

\begin{tabular}{lccc}
\hline Farmaco & $\begin{array}{c}\text { Costo acquisto per mg } \\
\text { (in euro) }\end{array}$ & $\begin{array}{c}\text { Dose/die } \\
\text { (mg) }\end{array}$ & $\begin{array}{c}\text { Costo me dio die } \\
\text { (in euro) }\end{array}$ \\
\hline Metadone & 0,013 & 100 & 1,30 \\
Buprenorfina & 0,270 & 16 & 4,32 \\
\hline
\end{tabular}

\section{Tabella 5}

Costo medio giornaliero del farmaco (anno 2003)

\begin{tabular}{lccc}
\hline & $\begin{array}{c}\text { Tempo medio } \\
\text { in minuti }\end{array}$ & $\begin{array}{c}\text { Costo medio } \\
\text { del personale } \\
\text { (in euro) }\end{array}$ & $\begin{array}{c}\text { Costo medio totale } \\
\text { per somministrazione } \\
\text { (in euro) }\end{array}$ \\
\hline $\begin{array}{l}\text { Metadone } \\
\text { - medico }\end{array}$ & 5 & 3,28 & 4,78 \\
- infermiere & 5 & 1,50 & \\
Buprenorfina & & & \\
- medico & 5 & 3,28 & 6,28 \\
- infermiere & 10 & 2,99 & \\
\hline
\end{tabular}

Tabella 6

Tempi e costi medi per somministrazione

\section{Costo di acquisto dei farmaci}

Il costo di acquisto per "mg" dei farmaci somministrati è stato ricavato sulla base del prezzo effettivo d'acquisto delle farmacie di alcuni Ser.T. distribuiti sul territorio nazionale ${ }^{\mathrm{ii}}$ [13]. Si è infatti ritenuto non sufficiente l'applicazione al prezzo al pubblico (Informatore Farmaceutico anno 2003) dello sconto minimo (50\%) previsto dalla legge (ex. L.386/75). Tale procedura, peraltro comunemente applicata, può non rispecchiare la realtà nel caso di prodotti, come gli oppioidi, per i quali lo sconto applicato può essere superiore al $50 \%$. Si è quindi chiesto ad alcuni Ser.T. di rilevare il prezzo di acquisto da parte della propria farmacia con particolare riferimento a metadone. Si è così determinato il costo medio effettivo di acquisto per confezione di ognuno dei due principi attivi considerati. Come si può vedere, per entrambi i prodotti lo sconto è superiore al 50\% (Tabella 4).

Per calcolare il costo medio giornaliero del farmaco sono stati presi in considerazione i dosaggi $16 \mathrm{mg}$ per buprenorfina e $100 \mathrm{mg}$ per metadone [14-18-21-23] (Tabella 5).

\section{Costo delle somministrazioni}

Per ogni singola somministrazione effettuata nei Ser.T., oltre al costo di acquisto del farmaco, è stato necessario conteggiare anche il costo per le risorse assorbite dal personale sanitario (medici e infermieri) che partecipa alla somministrazione. In Tabella 6 viene indicato il tempo medio, espresso in minuti, e il costo medio, espresso in euro, per ogni singola somministrazione per figura professionale che partecipa alla procedura [19, 20].

\section{Costo per assenza dal lavoro}

I soggetti che assumono buprenorfina o metadone perdono giornalmente una certa quantità del proprio tempo per recarsi ai Ser.T. per ricevere le terapia. In base alla numerosità e alla distribuzione territoriale dei Ser.T. si è stimato che mediamente un soggetto possa coprire le distanze di andata e ritorno dalla struttura in circa 45 minuti, e che permanga all' interno di essa, compresa l'eventuale attesa, mediamente di 15 minuti, con una perdita complessiva per ogni somministrazione di circa un'ora.

Per la determinazione degli svantaggi in termini di assenza lavorativa si è utilizzato il metodo del capitale umano [8]. In questa ottica ad ogni ora di assenza dal lavoro dovuta alla somministrazione della terapia viene attribuito un valore corrispondente a quello della produzione perduta. Per la determinazione del costo 


\begin{tabular}{lccc}
\hline & $\begin{array}{c}\text { Reddito lordo } \\
\text { annuo (in euro) }\end{array}$ & $\begin{array}{c}\text { Giorni lavorativi } \\
\text { medi annui (gg) }\end{array}$ & $\begin{array}{c}\text { Costo medio } \\
\text { per ora (in euro) }\end{array}$ \\
\hline $\begin{array}{l}\text { Costo medio per } \\
\text { ora lavorativa persa }\end{array}$ & $28.810,03$ & 220 & 16,37 \\
\hline
\end{tabular}

\section{Tabella 7}

Costo medio per ora lavorativa persa

di un'ora di lavoro persa si è considerato il reddito da lavoro per unità di lavoro dipendente per attività economica (fonte ISTAT) diviso per i 220 giorni lavorativi medi annui (Tabella 7).

\section{Descrizione dell'analisi di sensibilità}

Per valutare la robustezza dei risultati ottenuti è stata condotta un'analisi di sensibilità $[8,10]$ a due vie (two-way) sulla "dose media giornaliera" e un' analisi di sensibilità a una via (one-way) sui seguenti parametri: "probabilità dei soggetti trattati in funzione della durata

\section{Analisi di sensibilità}

L'analisi di sensibilità a due vie è stata condotta facendo variare simultaneamente i dosaggi di buprenorfina tra gli 8$32 \mathrm{mg} / \mathrm{die}$ e di metadone tra i 60-120 mg/die. L'analisi di sensibilità ad una via è stata invece eseguita facendo variare i valori del $+/-10 \%$ dei seguenti parametri: "probabilità dei soggetti trattati in funzione della durata della terapia", "probabilità secondo il numero di somministrazioni settimanali", "costo medio di acquisto dei trattamenti per mg", "tempi e costi medi per somministrazione" e "costo medio per ora lavorativa persa". In nessun caso si è riscontrata una sensibile variazione dei risultati rispetto ai valori di base. È stata inoltre testata la possibilità di realizzare l'affido a metadone a partire da 3 mesi; anche in questo caso non si è ri-

\begin{tabular}{|c|c|c|c|}
\hline Trattamento & $\begin{array}{l}\text { Costo Medio Totale } \\
\text { (12 mesi) (in euro) }\end{array}$ & $\begin{array}{l}\text { Costo Medio Sanitario } \\
\text { (12 mesi) (in euro) }\end{array}$ & $\begin{array}{c}\text { Costo Medio } \\
\text { Perdita Produttività (Indiretto) } \\
\text { (12 mesi) (in euro) }\end{array}$ \\
\hline Metadone & $\begin{array}{c}5.151,22 \\
100 \%\end{array}$ & $\begin{array}{c}1.429,69 \\
28 \%\end{array}$ & $\begin{array}{c}3.721,53 \\
72 \%\end{array}$ \\
\hline Buprenorfina & $\begin{array}{c}4.326,80 \\
100 \%\end{array}$ & $\begin{array}{c}1.901,79 \\
44 \%\end{array}$ & $\begin{array}{c}2.425,01 \\
56 \%\end{array}$ \\
\hline
\end{tabular}

Tabella 8

Costo medio totale (sanitario + perdita produttività) per trattamento (12 mesi anno 2003) della terapia", "probabilità secondo il numero di somministrazioni settimanali", "costo medio di acquisto dei trattamenti per mg", "tempi e costi medi per somministrazione" e "costo medio per ora lavorativa persa”.

\section{RISULTATI}

\section{Costi medi di trattamento}

In Tabella 8 sono riportati in sintesi i costi medi totali, diretti e indiretti per le due alternative di trattamento considerate lungo un orizzonte temporale di un anno.

Il costo medio totale per un soggetto tossicodipendente trattato con buprenorfina è di $4.326,80$ euro, inferiore di 824,42 euro rispetto all'alternativa rappresentata da metadone (5.151,22 euro). I costi medi sanitari del trattamento con metadone sono inferiori di 490,09 euro rispetto al trattamento con buprenorfina (1.901,79 euro per Bpn e 1.429,65 euro per Met), mentre i costi medi indiretti mostrano un significativo minor costo a favore di buprenorfina di 1.296,52 euro (2.425,01 euro per Bpn e $3.721,53$ euro per Met).

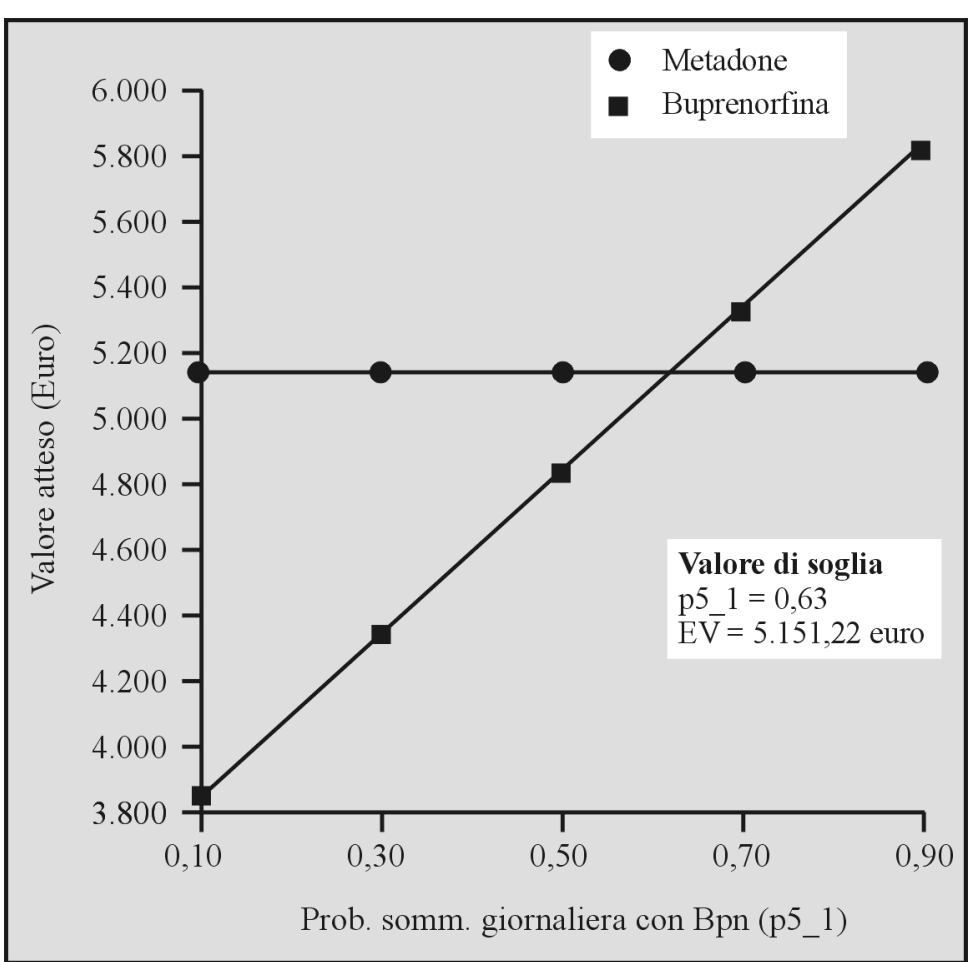

Figura 2

Analisi di sensibilità per la probabilità di somministrazione giornaliera per buprenorfina nel medio e lungo termine 
scontrata una variazione sensibile dei risultati rispetto al caso base.

Nella Figura 2 viene mostrata l'analisi di sensibilità per la probabilità di somministrazione giornaliera per buprenorfina nel medio e lungo termine; il modello presenta un punto di soglia per una probabilità di somministrazione giornaliera al $63 \%$ dei pazienti, ossia esiste un punto di equivalenza tra le due strategie quando la probabilità di somministrazione giornaliera per buprenorfina avviene nel $63 \%$ dei pazienti. Questo significa che la convenienza della strategia buprenorfina cresce all' aumentare della probabilità di somministrazione settimanale del farmaco.

Nella Figura 3 viene invece mostrata l'analisi di sensibilità per il costo orario dell' attività lavorativa perduta. Il modello presenta un valore soglia pari a 6,00 euro equivalente circa a $23 \mathrm{mn}$ lavorativi persi per i trasferimenti e l'assunzione della terapia, ossia per tale valore il modello proposto presenta un' equivalenza di costo atteso tra le due strategie.

Si è infine ipotizzato di estendere l'orizzonte temporale di analisi fino a 24 mesi. In questo caso, il costo medio totale per un soggetto tossicodipendente trattato con buprenorfina è risultato pari a 6.538,66 euro vs. 8.404,10 euro di metadone, con un risparmio maggiore in termini percentuali rispetto a quanto emerso nell'analisi a 12 mesi.

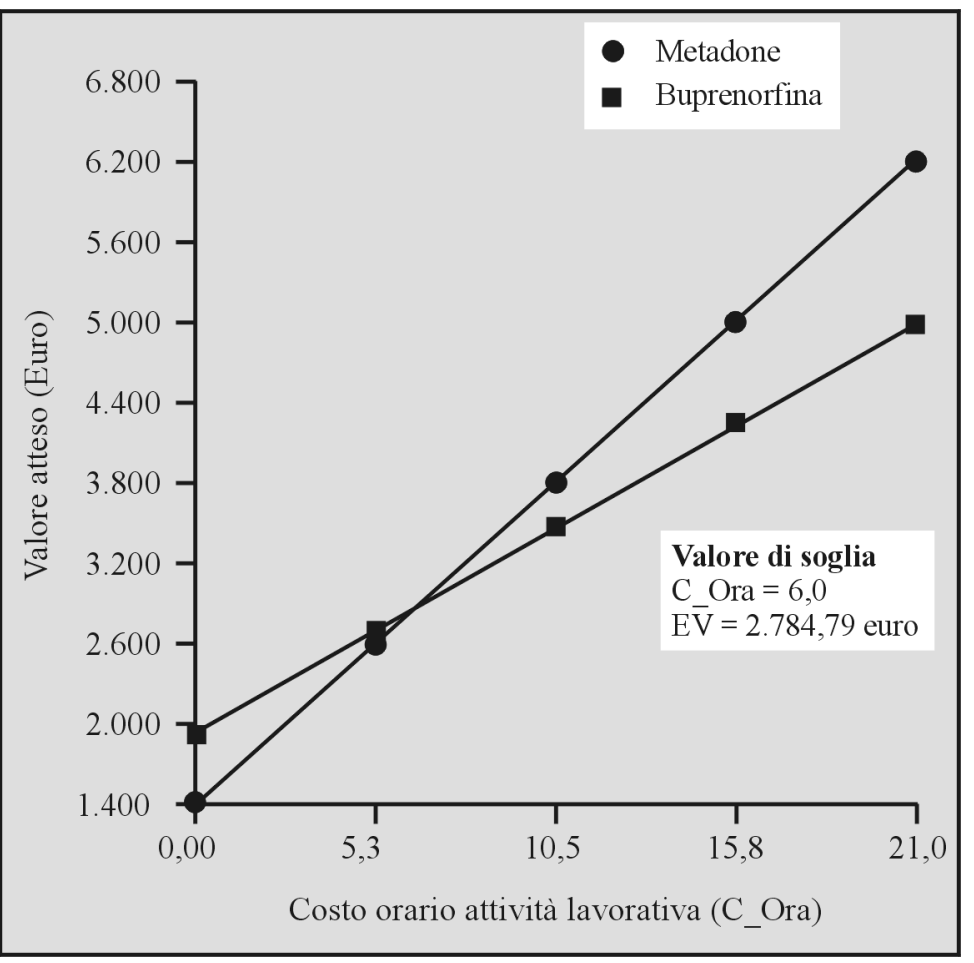

Figura 3

Analisi di sensibilità per il costo orario dell'attività lavorativa perduta

\section{DISCUSSIONEECONCLUSIONE}

Questa analisi farmacoeconomica è stata condotta con l'obiettivo di valutare i costi di trattamento di buprenorfina ( $16 \mathrm{mg} / \mathrm{die})$ versus metadone (100 mg/die) nei soggetti tossicodipendenti.

Un primo limite di questa analisi è dovuto al fatto che per alcune voci di probabilità di accadimento, a causa dell'incompletezza dei dati riportati dalle fonti cui si è potuto fare riferimento in fase di analisi, sono state fatte delle stime. Un secondo limite deriva dal fatto di non avere calcolato l'efficacia dei due trattamenti, in quanto al momento attuale nella realtà italiana non sono ancora emersi elementi che connotino la superiorità clinica di uno dei due principi attivi tali da giustificarne la preferenza. Proprio per questo motivo, la ricerca clinica sta indagando l'eventuale presenza di fattori predittivi di performance terapeutica che possano favorire l'orientamento verso uno o l'altro trattamento, soprattutto in termini di qualità di vita del paziente. Tra i costi degli operatori sanitari presenti in un Ser.T. non sono stati considerati nel modello adottato quelli relativi agli psicologi, agli assistenti sociali e agli educatori professionali, in quanto ugualmente presenti in entrambi i trattamenti.

Nella valutazione economica, inoltre, non è stato preso in considerazione il costo delle analisi di laboratorio per la ricerca delle sostanze di abuso, test di norma, sia nei pazienti trattati con buprenorfina sia in quelli trattati con metadone. Secondo quanto calcolato in questa analisi di minimizzazione dei costi, trattare un paziente con buprenorfina permette di risparmiare mediamente 824,42 euro rispetto all'alternativa di trattamento con metadone.

Questo risultato è spiegabile soprattutto alla luce dei maggiori costi indiretti, dovuti alla perdita di ore lavorative, associati a metadone; infatti un paziente tossicodipendente trattato con metadone è costretto a recarsi al Ser.T. per ricevere la somministrazione del farmaco con una maggior frequenza settimanale di quanto non venga fatto da un soggetto trattato con buprenorfina, che a fronte della possibilità di ricevere la somministrazione del trattamento a dosaggi multipli riduce il numero di contatti settimanali con il Ser.T. L'analisi di sensibilità condotta su tale parametro ha mostrato un valore di soglia a 6,00 euro, valore minimo per il quale le due alternative presentano il medesimo costo atteso.

Per quanto riguarda invece i costi diretti è da notare che il più alto costo di acquisto per $\mathrm{mg}$ sostenuto per il trattamento farmacologico dei pazienti con buprenorfina ( $\mathrm{Bpn}$ : 0,270 euro per mg vs. Met: 0,013 euro per $\mathrm{mg}$ ) risulta controbilanciato dal minor dosaggio giorna- 


\begin{tabular}{lcccc}
\hline & \multicolumn{2}{c}{ METADONE } & \multicolumn{2}{c}{ BUPRENORFINA } \\
& $\begin{array}{c}\text { No soggetti } \\
\text { trattati }\end{array}$ & $\begin{array}{c}\text { Costo Totale } \\
\text { (milioni di euro) }\end{array}$ & $\begin{array}{c}\text { No soggetti } \\
\text { trattati }\end{array}$ & $\begin{array}{c}\text { Costo Totale } \\
\text { (milioni di euro) }\end{array}$ \\
\hline Breve Termine & 13.000 & 8,7 & 16.000 & 12,7 \\
Medio Termine & 18.000 & 73,6 & 30.000 & 106 \\
Lungo Termine & 47.000 & 320,6 & 32.000 & 219,3 \\
TOTALE & 78.000 & 402 & 78.000 & 338 \\
\hline
\end{tabular}

liero (Bpn: 16mg vs Met: 100mg) e dal minor numero di somministrazioni settimanali (costo personale medico e infermieristico). Il costo sanitario sul totale del costo atteso, nelle due strategie considerate, è risultato compreso tra il 28\% (Met) e il 44\% (Bpn), mentre le perdite di produzione sono risultate rilevanti soprattutto nella coorte metadone in quanto, da quello dimostrato in precedenza dalle statistiche, il trattamento con metadone prevede un programma terapeutico più a lungo termine $(23 \%$ nel medio e $60 \%$ nel lungo termine).

In tutte le analisi di sensibilità, sia a due che a una via, buprenorfina, anche nelle ipotesi più sfavorevoli, ha mostrato di essere costsaving rispetto a metadone. Questo ci permette di confermare la robustezza dei risultati trovati. Il modello è risultato sensibile alla probabilità di somministrazione 3 volte la settimana di buprenorfina: ossia, con una probabilità superiore al $38 \%$ di somministrazione settimanale di buprenorfina nel medio e lungo termine, questa strategia è risultata meno costosa, sulla base delle ipotesi proposte, rispetto a metadone. Il modello è stato inoltre sviluppato in termini conservativi per quanto riguarda l'alternativa buprenorfina. Non sono stati considerati ad esempio i possibili miglioramenti in termini di qualità di vita che buprenorfina comporta [22], e non è stata considerata la possibilità di realizzare un parziale affido con buprenorfina o di passare alla terapia a giorni alterni a partire anche da 15 giorni.

L'importanza del minor costo medio di trattamento di buprenorfina rispetto a metadone è ancora più evidente se si considera l'impatto economico che ha la dipendenza da eroina per l'intera società. Infatti valutando i dati nazionali dell' anno 2001 si ricava che il numero di utenti dei Ser.T. ammonta a circa 150.000, di cui 123.000 eroinodipendenti. Metadone è stato adottato per circa 78.000 di essi (13.000 nel breve, 18.000 nel medio e 47.000 nel lungo termine), mentre sono stati trattati solo circa 5.000 soggetti con buprenorfina (1.000 nel breve, 1.920 nel medio e 2.080 nel lungo termine). Facendo una ulteriore simulazione sulla base dei dati di costo determinati dal modello costruito ad hoc per questa valutazione economica, nel 2001 i 78.000 soggetti eroinodipendenti curati con metadone sarebbero costati circa 402 milioni di euro, mentre se fossero stati trattati con buprenorfina sarebbero costati 338 milioni di euro (Tabella 9).

I risultati dello studio, quindi, mostrano come, a parità di efficacia e nonostante un maggior costo di acquisto, buprenorfina, in virtù di trattamenti meno prolungati e soprattutto di regimi somministrativi più flessibili che consentono un minor numero di contatti fra pazienti e personale dei Ser.T., sia l'alternativa a minor costo, dal punto di vista della Società, rispetto a metadone nel trattare i pazienti tossicodipendenti.

Questo studio è stato reso possibile da un finanziamento messo a disposizione da Essex Italia S.p.a.

Si ringrazia la dottoressa Chiara Del Bo per la collaborazione alla redazione del presente lavoro.

\section{Tabella 9}

Simulazione costi totali anno 2001 trattamento eroinodipendenza

\section{BIBLIOGRAFIA}

1. Lucchini A (a cura di). L'uso della Buprenorfina nel trattamento della tossicodipendenza. Clinica delle dipendenze e dei comportamenti di abuso/Quaderni, FrancoAngeli 2002.

2. Cornish JW, Metzger D, Woody GE, Wilson D, McLellan AT, Vandergrift B, CTBrien CP. Naltrexone pharmacotherapy for opioid dependent federal probationers, J. Subst. Abuse Treat. 1997; 14, 529-534.

3. Oda Y, Kharasch ED. Metabolism of methadone and levo-alpha-acetylmethadol (LAAM) by human intestinal cytochrome P4503A4 (CYP3A4): potential contribution of intestinal metabolism to presynaptic clearance and bioactivation" J. Pharmacol. Exp. Ther. 2001; 298, 1021-1032. 
Analisi dei costi di buprenorfina vs metadone nella terapia dei soggetti con dipendenza da oppiacei

4. Moody DE, Alburges ME, Parker RJ, Collins JM, Strong JM. The involment of cytochrome P450 in the Ndemethylation of L-alpha-acetylmethadol (LAAM), nor-LAAM, and methadone. Drug Metab. Dispos. 1997;25: 1347-1353.

5. Novick DM, Richman BL, Friedman JM, Friedman JE et al. The medical status of methadone maintained in the treatment for 11-18 years. Drug Alcohol Depend. 1993; 33: 235-245.

6. Ibrahim RB, Wilson JG, Thorsby ME, Edwards DJ. Effects of buprenorphine on CYP3A activity in rat and human liver microsomes. Life Sci. 2000; 66: 1293-1298.

7. Walsh SL, Preston KL, Stitzer ML et al. Clinical pharmacology of buprenorphine: ceiling effect at high doses. Clin Pharmacol Ther 1994; 55: 569-580.

8. Drummond MF, O’Brien BJ, Stoddart GL, Torrance GW. (Edizione italiana a cura di Fattore G, Garattini L, Lucioni C) Metodi per la valutazione economica dei programmi sanitari. Il Pensiero Scientifico Editore, 2000.

9. West SL, O’Neal KK, Graham CW. A meta-analysis comparing the effectiveness of buprenorphine and methadone. J. Subst. Abuse 2000; 12: 405-414.

10. Weinstein MC, Fineberg HV. L'analisi della decisione in medicina clinica. Fondazione Smith Kline FrancoAngeli 1984.

11. O'Connor PG, Oliveto AH, Shi JM, Triffleman E, Carroll KM, Kosten TR, Rounsaville BJ. A pilot study of primary care based buprenorphine maintenance for heroin dependence, Am.J. Drug Alcohol Abuse 1996: 22, 523-531

12. Ministero della Salute - Dipartimento della tutela della salute umana, della sanità pubblica veterinaria e dei rapporti internazionali - Direzione Generale della Prevenzione - Ufficio VI. Promozione dei comportamenti e stili di vita per la salute e relativi interventi in materia di dipendenza da farmaci e sostanze di abuso e di AIDS; sanità penitenziaria. Rilevazione attività nel settore tossicodipendenze - anno 2001 - Roma, ottobre 2002.

13. Lucioni C, Ravasio R, Concia E. Terapia empirica con antibiotici parenterali in pazienti ospedalizzati affetti da infezioni alle basse vie respiratorie: una valutazione farmacoeconomica. PharmacoEconomics Italian Research Articles 2001: vol. 3 (1); 37-47.

14. Caplehorn RM, Bell J. Methadone dosage and retention of patients in maintenance treatment. Medical Journal of Australia 1999 154: 195-199.

15. Donny EC, Walsh SL, Bigelow GE, Eissenberg T, Stitzer ML. High-dose methadone produce superior opioid blockade and comparable withdrawal suppression to lower doses in opioid-dependent humans, Psychoph. 2002 161, 202212.

16. Hartel DM et al. Heroin use during methadone maintenance treatment: the importance of methadone dose and cocaine use. Am. J. Public Health 1995; 85: 83-88.

17. Fudala PJ et al. Use of Buprenorphine in the treatment of opioid addiction II. Physiologic and behavioural effects of daily and alternate-day administration and abrupt withdrawal. Clin Pharm Ther 1990 47: 525-534.

18. Schottenfeld RS et al. Thrice-weekly versus daily buprenorphine maintenance. Society of Biological Psychiatry 2000; 47: 1072-1079.

19. D’Egidio P. I costi dei programmi di trattamento per l'abuso di sostanze. Giugno 2002.

20. Calculating the social cost of illicit drugs. Council of Europe publishing 2001.

21. Johnson RE, Chutuape MA, Strain C, Walsh SL, Stitzer ML, Bigelow GE. A comparison of levometadil acetate, buprenorphine, and methadone for opioid dependence. N. Eng. J. Med. 343(18): 1290-1297, 2000.

22. Barnett PG, Zaric GS. The cost-effectiveness of buprenorphine maintenance therapy for opiate addiction in US", addiction (2001) 96, 1267 - 1278

23. Zubieta $\mathrm{JK}$ et al. Buprenorphine-induced changes in mu-opioid receptor availability in male heroin-dependent volunteers: a preliminary study. Neuropsychopharm. 23 (3): 326-334, 2000. 